\title{
Role of Defect Density in Optimizing MWCNT-Polymer Composite Sensor Performance: Tuning of Its Acceptable Limit by Acid Treatment Condition
}

\author{
Authors: Sakshi Sharma; S. S. Islam; Poonam Sehrawat; Sukhvir Singh \\ Source: Science of Advanced Materials, Volume 7, Number 7, July 2015, pp. 1341-1350(10) \\ Publisher: American Scientific Publishers
}

\begin{abstract}
:
Surface defects of carbon nanotubes (CNTs) are always a debatable issue about its role in sensing properties and a systematic study is presented in this paper for its exploration. Intentional defect sites of various types were produced at different dose by liquid phase oxidation technique followed by acid reflux for different durations; and its effect on the toxic gas sensing performances were experimented in a rigorous manner to understand the actual sensing mechanism in the presence of increasing dose of defects. Interestingly, the sensor response was found to increase to a certain limit, dependent on the critical acid treatment time, beyond which the performance of the sensor goes reverse. And in depth analysis has been put up for the first time to optimize the acid treatment condition in order to achieve the maximum sensitivity of the sensor. Transmission electron microscopy (TEM) results confirm the introduction of many new types of defects on Multi-walled carbon nanotubes (MWCNTs) surface as acid treatment was intensified. Its ultimate consequence is that the controllably introduction of desired density of defects on nanotubes, by tuning acid treatment condition, plays another major role to improve the gas sensor sensitivity apart from the conventional techniques where increase in surface area by stable homogenous dispersion of CNTs is prime concern.
\end{abstract}

Keywords: COMPOSITE; DEFECT DENSITY; GAS SENSOR; MWCNTS

Document Type: Research Article

DOI: http://dx.doi.org/10.1166/sam.2015.2050

Publication date: July 1, 2015 